\title{
Associação entre vitimização por violência entre parceiros íntimos e depressão em adultos brasileiros
}

\author{
The association between victimization resulting from intimate \\ partner violence and depression among Brazilian adults
}

Aline Natália Silva (https://orcid.org/0000-0002-1811-3703) ${ }^{1}$

Catarina Machado Azeredo (https://orcid.org/0000-0002-6189-4429) ${ }^{1}$

${ }^{1}$ Faculdade de Medicina, Universidade Federal de Uberlândia. Av. Pará 1720/ bloco $2 \mathrm{U}$, Umuarama. 38400-902 Uberlândia MG Brasil.catarina.azeredo@ ufu.br

\begin{abstract}
The scope of this article was to assess the association between Intimate Partner Violence (IPV) and depression among Brazilian adults. This cross-sectional study used data from the 2013 National Health Survey with a representative sample of 49,025 Brazilian adults, with information on IPV, depression and socio-demographic conditions. For a description of characteristics associated with depression, including the core IPV data, analyses of simple and multiple logistic regression were performed. The analyses were performed using Stata 13.0 software. The prevalence of IPV was $1 \%$ in the general population, and $0.38 \%$ among men and $1.58 \%$ among women. The prevalence of depression was $3.94 \%$ (CI 95\% $=3.68-4.22)$ in the general population, $1.67 \%(C I$ 95\% $=1.40-1.94)$ among men and $6.03 \%(C I 95 \%=5.57-6.48)$ among women. Victims of IPV were more likely to report depression than non-victims $(a O R=2.90 ; C I 95 \%=$ 1.38-6.09). The conclusion drawn is that depression is associated with intimate partner victimization, which has important implications from a public health standpoint.
\end{abstract}

Key words Intimate partner violence, Depression, Prevalence, Cross-sectional studies
Resumo O objetivo do artigo foi avaliar a associação entre Violência por Parceiros Íntimos (VPI) e depressão em adultos brasileiros. Este estudo transversal utilizou dados da Pesquisa Nacional de Saúde (PNS) de 2013, com amostra representativa de 49.025 adultos brasileiros, com informações de VPI, depressão e condiçães sociodemográficas. Para a descrição de características associadas com a depressão, incluindo a exposição principal (VPI), foram realizadas análises de regressão logística simples e múltipla. As análises foram realizadas no programa Stata, versão 13.0. A prevalência de VPI foi de 1,0\% na população em geral, de 0,38\% entre homens e 1,58\% entre mulheres. A prevalência de depressão foi 3,94\% (IC95\% = 3,68-4,22) na população em geral, $e$ de 1,67\% (IC95\% = 1,40-1,94) entre os homens e 6,03\% (IC95\% = 5,57- 6,48) entre as mulheres. Vitimas de VPI tiveram mais chances de relatar depressão em relação às não vítimas ( $a O R=2,90$; IC95\% = 1,38-6,09). Concluiu-se que depressão está associada à VPI, o que tem implicações importantes no ponto de vista da saúde pública.

Palavras-chave Violência entre parceiros intimos, Depressão, Prevalência, Estudos transversais 


\section{Introdução}

A violência entre parceiros íntimos (VPI) é caracterizada como a violência física ou sexual, perseguição ou agressão psicológica vinda de um parceiro íntimo atual ou anterior ${ }^{1}$. $\mathrm{O}$ parceiro íntimo pode ser cônjuge, companheiro (a) ou namorado (a), não havendo a necessidade de relações sexuais, sendo que as relações podem ser emocionais e/ou afetivas'.

Esse tipo de violência constitui um grave problema de saúde pública tanto pelas altas prevalências quanto pelas graves consequências à saúde das vítimas ${ }^{2}$. Estudo realizado pela Organização Mundial de Saúde ${ }^{3}$ mostrou que a prevalência de VPI entre mulheres é elevada em vários países, com prevalência de $15 \%$ no Japão e $70 \%$ na Etiópia e no Peru, e prevalências mundiais que variam entre $29 \%$ e $62 \%$. Em capitais brasileiras, a prevalência de VPI foi cerca de $21,5 \%$ entre mulheres e $12,9 \%$ entre homens ${ }^{4}$. Várias são as consequências desenvolvidas pelas vítimas de VPI. Dentre os problemas psicológicos observados estão ansiedade, insônia, distúrbios sociais e depressão, que tem risco aumentado para os que sofrem esse tipo de violência ${ }^{5,6}$. Além dos problemas mentais, também há aqueles que afetam a saúde física das vítimas, como: traumas, fraturas, dor abdominal, dor de cabeça, dor lombar, distúrbios gastrointestinais, dentre outros ${ }^{7}$.

Ainda são poucos os estudos que englobam os homens como sendo vítimas nesse tipo de violência, pois a maioria dos textos coloca os homens nos papéis de agressores e perpetradores e as mulheres como sendo as vítimas ${ }^{8}$. Tendo em vista que o Brasil tem a maior população da América Latina e é um país de renda média ${ }^{9} \mathrm{com}$ elevada taxa de violência ${ }^{10}$ seria importante avaliar a associação entre VPI e depressão, de modo a subsidiar políticas de saúde que visem identificar vulnerabilidades entre as vítimas. Diante disso, o objetivo do presente trabalho é avaliar a associação entre VPI e depressão entre adultos brasileiros.

\section{Métodos}

\section{População de estudo, amostra e coleta de dados}

O presente estudo tem delineamento transversal e utilizou os dados da Pesquisa Nacional de Saúde (PNS) com amostra representativa de domicílios brasileiros, realizada em 2013. Os entrevistados foram selecionados por meio de amostragem em conglomerados em múltiplos estágios (setores censitários, domicílios particulares e indivíduos) ${ }^{11}$.

A população de base para a amostragem foi obtida pelo Censo de 2010, realizado pelo IBGE. Dos 81.767 domicílios selecionados, 62.986 foram entrevistados (78\% de taxa de resposta) e foram obtidos dados válidos de 60.202 que foram analisados no presente estudo (taxa de resposta final $=73,6 \%$ ). Após a seleção do domicílio, a seleção do morador respondente se deu de maneira aleatória entre os maiores de 18 anos de idade ${ }^{11}$. Para o presente estudo foram excluídos indivíduos com 60 anos ou mais o que resultou numa amostra final de 49.025 pessoas. Foram analisadas as respostas do questionário individual.

Para a coleta dos dados foram utilizados computadores de mão do tipo Personal Digital Assistance (PDA) pelos entrevistadores que passaram por um processo de treinamento para boa execução da atividade ${ }^{11}$. O projeto da PNS foi aprovado pela Comissão Nacional de Ética em Pesquisa - Conep, do Conselho Nacional de Saúde - CNS, em junho de $2013^{11}$.

\section{Variáveis}

\section{Desfecho}

A depressão foi considerada como desfecho da VPI. As perguntas sobre depressão incluíram relato de diagnóstico anterior ("Algum médico ou profissional de saúde mental (como psiquiatra ou psicólogo) já lhe deu diagnóstico de depressão?”) e procura ao serviço de saúde para atendimento ("Qual o principal motivo do(a) sr(a) não visitar o médico/serviço de saúde regularmente por causa da depressão?). Indivíduos que responderam não terem sido diagnosticados com depressão ou que apesar do diagnóstico relataram não procurarem atendimento por não estarem mais deprimidos foram considerados sem depressão. Considerou-se com depressão aqueles com diagnóstico e que responderam outros motivos, que não a cura, para não procurar por atendimento no serviço de saúde.

\section{Exposição de interesse principal}

A análise da VPI foi baseada nas seguintes questões: "Nos últimos meses, o(a) sr(a) sofreu alguma violência ou agressão de pessoa conhecida (como pai, mãe, filho(a), cônjuge, parceiro(a), namorado(a), amigo(a), vizinho(a))? Se a resposta a essa pergunta foi sim, prosseguiu-se com outra pergunta para identificar a relação exis- 
tente entre o agressor e a vítima. Indivíduos que responderam que a violência foi cometida por: "cônjuge, companheiro(a), namorado(a) e excônjuge, ex-companheiro(a), ex-namorado(a)" foram classificados como vítimas de VPI.

\section{Potenciais variáveis de confusão}

Foram consideradas possíveis variáveis de confusão: cor (branca, preta, amarela, parda e indígena), sexo, idade (em anos), escolaridade (ensino fundamental incompleto, fundamental completo, médio completo e superior completo), estado civil (com companheiro/ sem companheiro), a frequência no consumo de bebidas alcoólicas nos últimos 30 dias (nunca, menos de uma vez ao mês e mais de uma vez ao mês), e a procura por serviços de saúde que foi mensurada pelas seguintes perguntas ("Quantas vezes consultou o médico nos últimos 12 meses?" e "Nas duas últimas semanas, procurou algum lugar, serviço ou profissional de saúde para atendimento relacionado à própria saúde?”).

\section{Análise dos dados}

As análises descritivas foram realizadas considerando a ponderação para a estrutura amostral complexa, de modo a representar a população de adultos brasileiros.

Em uma primeira análise da associação entre depressão e as demais variáveis foram estimadas as prevalências de depressão em função de todas as variáveis do estudo, com o respectivo intervalo de confiança de 95\% (IC95\%) das prevalências. Da mesma forma, também foram analisadas a associação entre a violência entre parceiros íntimos e todas as outras variáveis. Foram descritos ainda os tipos de VPI e sua distribuição por sexo.

Para a descrição de características associadas à depressão, incluindo a exposição principal (VPI), foram realizadas análises de regressão logística simples e múltipla, uma vez que o desfecho do presente estudo era dicotômico (depressão: sim ou não).

A partir da revisão da literatura, selecionouse as variáveis consideradas potenciais confundidoras da associação entre VPI e depressão. A seleção das variáveis que seriam incluídas no modelo final seguiu o critério epidemiológico de confounding. A partir desse critério operacional, as variáveis que a partir do referencial teórico ${ }^{5} \mathrm{se}$ associavam tanto ao desfecho quanto à variável explicativa e não faziam parte da cadeia causal foram incluídas no modelo final para ajuste ${ }^{12}$. As variáveis $\operatorname{sexo}^{13}$, idade ${ }^{14}, \operatorname{cor}^{15}$, escolaridade ${ }^{4}$, estado civil ${ }^{14}$, consumo de álcool ${ }^{16}$ e procura por serviços de saúde ${ }^{6}$ cumpriram com esse critério. Realizou-se, então, análise bivariada entre cada uma destas variáveis e o desfecho, utilizando-se modelos de regressão logística simples. Foram obtidos odds ratios (OR) brutos e seus respectivos IC95\% a partir dessas análises. Posteriormente, foram obtidos os OR ajustados (aOR) por meio dos modelos múltiplos de regressão logística ajustados simultaneamente para todas as variáveis. A significância estatística foi assumida arbitrariamente como 5\%.

Todas as análises estatísticas foram realizadas no Software Stata SE versão 13.0 (StataCorp, Texas, 2009).

\section{Resultados}

Dentre os entrevistados, 1,0\% havia sofrido VPI. Dentre os homens, a prevalência de VPI foi $0,38 \%$ (IC95\% = 0,26-0,54) e entre mulheres, 1,58\% $(\mathrm{IC} 95 \%=1,36-1,82)$. Em relação à depressão, a prevalência foi de 3,94\% (IC95\% = 3,68-4,22), atingindo $1,67 \%($ IC95\% = 1,40-1,94) dos homens e 6,03\% (IC95\% = 5,57-6,48) das mulheres.

O perfil dos entrevistados pela PNS e caracterização de acordo com o diagnóstico de depressão pode ser observado na Tabela 1. A maioria das pessoas era do sexo feminino $(52,13 \%)$, se declarou de cor branca $(45,85 \%$; IC95\% $=45,21$ $46,49)$ e parda $(43,47 \%$; IC95\% $=42,82-44,10)$, possuía ensino fundamental completo $(71,87 \%$; IC95\% $=71,23-72,50)$ e relatou estar sem companheiro (59,92\%; IC95\% = 59,27-60,57). Dentre as pessoas que relataram ter depressão, observou-se maior prevalência entre as mulheres $(6,03 \%$; IC95\% = 5,57-6,48), com predomínio de cor branca $(4,64$; IC $95 \%=4,20-5,08)$, baixo nível de escolaridade $(6,70 \%$;IC95\% = 5,55-7,84, ensino fundamental incompleto), sem companheiro $(3,97 \%$; IC95\% = 3,62-4,62) e que não ingeriam bebidas alcoólicas $(5,00 \%$; IC95\% = 4,59-5,40).

As características em relação ao status de vitimização (sofreram ou não sofreram VPI) podem ser observadas na Tabela 2. Dentre as vítimas de VPI nota-se maior prevalência da violência entre mulheres $(1,58 \%$; IC95\% $=1,35-1,81)$, sem companheiro $(1,35 \%$ IC95\% $=1,14-1,55)$, com baixo nível de escolaridade (1,23\%; IC95\% = 1,04-1,41, ensino fundamental completo). A prevalência de depressão entre as vítimas de VPI foi de 12,17\% (IC95\% $=8,00-16,36)$, sendo superior à prevalência no total da amostra 3,94\% (IC95\% = 3,68$4,22)$. O tipo de violência mais relatado entre 


\begin{tabular}{|c|c|c|c|c|c|c|c|}
\hline \multirow{28}{*}{ 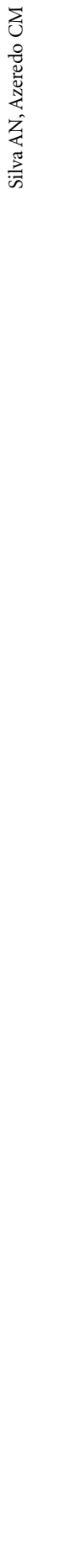 } & Tabela 1. Caracterís & ticas gerais $\mathrm{d}$ & amostra entre $c$ & $\mathrm{~s}$ indivíduos c & om e sem depre & ssão (PNS-20 & 13) \\
\hline & \multirow{2}{*}{ Características } & \multicolumn{4}{|c|}{ Depressão (\%) } & \multirow{2}{*}{ Total (\%) } & \multirow{2}{*}{ IC95\% } \\
\hline & & Sim $(\%)$ & IC95\% & Não (\%) & IC95\% & & \\
\hline & \multicolumn{7}{|l|}{ Sexo } \\
\hline & Masculino & 1,67 & $(1,40-1,94)$ & 98,33 & $(98,06-99,60)$ & 47,87 & $(47,87-47,87)$ \\
\hline & Feminino & 6,03 & $(5,57-6,48)$ & 93,97 & $(93,51-94,43)$ & 52,13 & $(52,13-52,13)$ \\
\hline & $\begin{array}{l}\text { Idade (média e } \\
\text { erro padrão) }\end{array}$ & $38,18(1,28)$ & $(37,93-38,43)$ & $36,98(0,34)$ & $(36,91-37,05)$ & $37,01(0,03)$ & $(36,95-37,07)$ \\
\hline & \multicolumn{7}{|l|}{ Cor } \\
\hline & Branca & 4,64 & $(4,20-5,08)$ & 95,36 & $(94,92-95,80)$ & 45,85 & $(45,21-46,49)$ \\
\hline & Preta & 2,81 & $(2,05-3,53)$ & 97,21 & $(96,46-97,95)$ & 9,35 & $(8,96-9,76)$ \\
\hline & Amarela & 1,98 & $(0,34-3,61)$ & 98,02 & $(96,39-99,65)$ & 0,86 & $(0,74-1,01)$ \\
\hline & Parda & 3,49 & $(3,09-3,87)$ & 96,51 & $(96,12-96,90)$ & 43,47 & $(42,82-44,10)$ \\
\hline & Indígena & 4,34 & $(1,07-7,99)$ & 95,46 & $(92,01-98,92)$ & 0,47 & $(0,40-0,56)$ \\
\hline & \multicolumn{7}{|l|}{ Estado civil } \\
\hline & $\begin{array}{l}\text { Com } \\
\text { companheiro }\end{array}$ & 3,90 & $(3,46-4,33)$ & 96,10 & $(95,66-96,54)$ & 40,08 & $(39,43-40,73)$ \\
\hline & $\begin{array}{l}\text { Sem } \\
\text { companheiro }\end{array}$ & 3,97 & $(3,62-4,62)$ & 96,03 & $(95,68-96,38)$ & 59,92 & $(59,27-60,57)$ \\
\hline & \multicolumn{7}{|l|}{ Escolaridade } \\
\hline & $\begin{array}{l}\text { Fundamental } \\
\text { incompleto }\end{array}$ & 6,70 & $(5,55-7,84)$ & 93,30 & $(92,16-94,45)$ & 9,82 & $(9,43-10,22)$ \\
\hline & $\begin{array}{l}\text { Fundamental } \\
\text { completo }\end{array}$ & 3,58 & $(3,24-3,91)$ & 96,42 & $(96,08-96,76)$ & 71,87 & $(71,23-72,50)$ \\
\hline & $\begin{array}{l}\text { Médio } \\
\text { completo }\end{array}$ & 3,96 & $(3,28-4,63)$ & 96,04 & $(95,37-96,72)$ & 17,50 & $(16,95-18,07)$ \\
\hline & $\begin{array}{l}\text { Superior } \\
\text { completo }\end{array}$ & 4,01 & $(1,56-4,63)$ & 95,99 & $(93,54-98,44)$ & 0,81 & $(0,69-0,94)$ \\
\hline & \multicolumn{7}{|l|}{$\begin{array}{l}\text { Consumo de } \\
\text { álcool }\end{array}$} \\
\hline & Nunca & 5,00 & $(4,59-5,40)$ & 95,00 & $(94,59-95,41)$ & 55,37 & $(54,70-56,05)$ \\
\hline & $\begin{array}{l}\text { Menos de uma } \\
\text { vez ao mês }\end{array}$ & 3,22 & $(2,54-3,89)$ & 96,78 & $(96,10-97,46)$ & 15,21 & $(14,70-15,73)$ \\
\hline & $\begin{array}{l}\text { Mais de uma } \\
\text { vez ao mês }\end{array}$ & 2,33 & $(1,95-2,71)$ & 97,67 & $(97,29-98,05)$ & 29,42 & $(28,79-30,06)$ \\
\hline & \multicolumn{7}{|l|}{$\begin{array}{l}\text { Procura por } \\
\text { serviços de saúde }\end{array}$} \\
\hline & $\begin{array}{l}\text { Últimos } \\
12 \text { meses } \\
\text { (médico) }\end{array}$ & 6,11 & $(5,65-6,57)$ & 93,89 & $(93,43-94,35)$ & 69,59 & $(68,81-70,39)$ \\
\hline & $\begin{array}{l}\text { Últimas } 2 \\
\text { semanas }\end{array}$ & 10,06 & $(8,99-11,14)$ & 89,94 & $(89,94-91,01)$ & 15,98 & $(15,48-16,51)$ \\
\hline
\end{tabular}

as vítimas foi a violência psicológica $(49,73 \%$; IC95\% $=46,29-53,17)$ seguida pela violência física $(42,69 \%$; IC95\% = 39,19-46,25), esses dois tipos de violência foram predominantes em ambos os sexos (Tabela 3).

A Tabela 4 apresenta os resultados dos modelos bruto e ajustado para depressão. Comparando-se os modelos bruto e ajustado, observa-se uma pequena redução no OR de depressão entre pessoas vítimas de VPI após o ajuste, mas ainda assim a vitimização esteve positivamente associada à depressão, tendo as vítimas de VPI uma probabilidade 2,90 vezes maior de relatar depressão em relação as pessoas que não foram vítimas de VPI $(\mathrm{aOR}=2,90$; IC95\% = 1,38-6,09). Observase ainda que as mulheres e pessoas de cor branca tiveram maior probabilidade de referir depressão. Em relação ao estado civil, as pessoas sem 
Tabela 2. Características gerais da amostra entre os indivíduos que sofreram e não sofreram VPI (PNS2013).

\begin{tabular}{|c|c|c|c|c|}
\hline Características & Sofreu VPI (\%) & IC95\% & $\begin{array}{c}\text { Não sofreu VPI } \\
(\%)\end{array}$ & IC95\% \\
\hline \multicolumn{5}{|l|}{ Sexo } \\
\hline Masculino & 0,38 & $(0,24-0,52)$ & 99,62 & $(99,48-99,75)$ \\
\hline Feminino & 1,58 & $(1,35-1,81)$ & 98,42 & $(98,19-98,65)$ \\
\hline Idade (média e erro padrão) & $36,8(0,26)$ & $(36,3-37,3)$ & $37,0(0,03)$ & $(36,9-37,1)$ \\
\hline \multicolumn{5}{|l|}{ Cor } \\
\hline Branca & 0,73 & $(0,55-0,90)$ & 99,27 & $(99,09-99,44)$ \\
\hline Preta & 1,55 & $(1,00-2,10)$ & 98,45 & $(97,90-99,01)$ \\
\hline Amarela & 0,13 & $(0,00-0,33)$ & 99,87 & $(99,67-100,01)$ \\
\hline Parda & 1,19 & $(0,97-1,41)$ & 98,81 & $(98,60-99,03)$ \\
\hline Indígena & 2,49 & $(0,00-6,39)$ & 97,51 & $(93,61-100,01)$ \\
\hline \multicolumn{5}{|l|}{ Estado civil } \\
\hline Com companheiro & 0,50 & $(0,35-0,65)$ & 99,5 & $(99,35-99,65)$ \\
\hline Sem companheiro & 1,35 & $(1,14-1,55)$ & 98,65 & $(98,44-98,86)$ \\
\hline \multicolumn{5}{|l|}{ Escolaridade } \\
\hline Fundamental incompleto & 0,41 & $(0,19-0,62)$ & 99,59 & $(99,38-99,80)$ \\
\hline Fundamental completo & 1,23 & $(1,04-1,41)$ & 98,77 & $(98,59-98,96)$ \\
\hline Médio completo & 0,95 & $(0,56-1,33)$ & 99,05 & $(98,67-99,44)$ \\
\hline Superior completo & 0,16 & $(0,00-1,13)$ & 99,84 & $(99,52-100,01)$ \\
\hline \multicolumn{5}{|l|}{ Consumo de álcool } \\
\hline Nunca & 0,97 & $(0,79-1,15)$ & 99,03 & $(98,84-99,21)$ \\
\hline Menos de uma vez ao mês & 1,13 & $(0,82-1,43)$ & 98,87 & $(98,57-99,18)$ \\
\hline Mais de uma vez ao mês & 1,01 & $(0,74-1,28)$ & 98,99 & $(98,71-99,26)$ \\
\hline \multicolumn{5}{|l|}{ Procura por serviços de saúde } \\
\hline Últimos 12 meses (médico) & 1,12 & $(0,92-1,32)$ & 98,88 & $(98,67-99,08)$ \\
\hline Últimas 2 semanas & 1,28 & $(0,92-1,64)$ & 98,72 & $(98,36-99,08$ \\
\hline Depressão & 12,17 & $(8,00-16,36)$ & 3,7 & $(3,42-3,96)$ \\
\hline
\end{tabular}

Tabela 3. Tipo de violência por parceiro íntimo em relação ao sexo da vítima (PNS-2013).

\begin{tabular}{|c|c|c|c|c|c|c|}
\hline \multirow{2}{*}{$\begin{array}{c}\text { Tipo de } \\
\text { violência }\end{array}$} & \multicolumn{4}{|c|}{ Sexo (\%) } & \multirow{2}{*}{ Total (\%) } & \multirow{2}{*}{ IC95\% } \\
\hline & Masculino(\%) & IC95\% & Feminino (\%) & IC95\% & & \\
\hline Física & 40,8 & $(35,29-46,37)$ & 44,28 & $(40,12-48,53)$ & 42,69 & $(39,19-46,25)$ \\
\hline Sexual & 0 & não observado & 0,46 & $(0,19-1,07)$ & 0,25 & $(0,11-0,58)$ \\
\hline Psicológica & 52,54 & $(46,91-58,11)$ & 47,32 & $(43,17-51,5)$ & 49,73 & $(46,29-53,17)$ \\
\hline Outros & 6,65 & $(4,79-9,14)$ & 7,92 & $(6,11-10,22)$ & 7,34 & $(5,94-9,04)$ \\
\hline
\end{tabular}

companheiro tiveram maior probabilidade de apresentar depressão. No que se refere ao acesso aos serviços de saúde, observou-se que as pessoas que procuraram por atendimento relataram ter mais depressão.

\section{Discussão}

O presente estudo analisou a associação entre VPI e depressão no Brasil, e os resultados mostraram que as vítimas de VPI tiveram maior pro- 
Tabela 4. Modelo de regressão logística simples e múltipla de variáveis associadas à depressão (PNS-2013).

\begin{tabular}{|c|c|c|}
\hline Variáveis & $\begin{array}{l}\text { Modelo bruto } \\
\text { O.R(IC 95\%) }\end{array}$ & $\begin{array}{c}\text { Modelo } \\
\text { ajustado } \\
\text { a O.R(IC } 95 \%) \\
\end{array}$ \\
\hline \multicolumn{3}{|l|}{ Sexo } \\
\hline Masculino & 1 & 1 \\
\hline Feminino & $3,77(3,14-4,53)$ & $2,44(1,64-3,63)$ \\
\hline Idade (anos) & $1,04(1,03-1,04)$ & $1,03(1,03-1,04)$ \\
\hline \multicolumn{3}{|l|}{ Cor } \\
\hline Branca & 1 & 1 \\
\hline Preta & $0,59(0,44-0,79)$ & $0,55(0,35-0,86)$ \\
\hline Amarela & $0,41(0,18-0,97)$ & $0,51(0,13-1,95)$ \\
\hline Parda & $0,74(0,63-0,86)$ & $0,75(0,57-0,99)$ \\
\hline Indígena & $0,97(0,44-2,18)$ & $0,66(0,12-3,66)$ \\
\hline \multicolumn{3}{|l|}{ Estado civil } \\
\hline $\begin{array}{l}\text { Com } \\
\text { companheiro }\end{array}$ & 1 & 1 \\
\hline Sem companheiro & $1,02(0,88-1,18)$ & $1,40(1,08-1,82)$ \\
\hline \multicolumn{3}{|l|}{ Escolaridade } \\
\hline $\begin{array}{l}\text { Fundamental } \\
\text { incompleto }\end{array}$ & 1 & 1 \\
\hline $\begin{array}{l}\text { Fundamental } \\
\text { completo }\end{array}$ & $0,52(0,41-0,66)$ & $0,96(0,68-1,37)$ \\
\hline Médio completo & $0,57(0,43-0,76)$ & $0,77(0,51-1,16)$ \\
\hline $\begin{array}{l}\text { Superior } \\
\text { completo }\end{array}$ & $0,57(0,27-1,18)$ & $0,78(0,32-1,93)$ \\
\hline \multicolumn{3}{|l|}{ Consumo de álcool } \\
\hline Nunca & 1 & 1 \\
\hline $\begin{array}{l}\text { Menos de uma } \\
\text { vez ao mês }\end{array}$ & $0,63(0,50-0,80)$ & $0,84(0,56-1,27)$ \\
\hline $\begin{array}{l}\text { Mais de uma vez } \\
\text { ao mês }\end{array}$ & $0,45(0,37-0,55)$ & $0,72(0,49-1,03)$ \\
\hline \multicolumn{3}{|l|}{$\begin{array}{l}\text { Procura por serviços } \\
\text { de saúde }\end{array}$} \\
\hline $\begin{array}{l}\text { Últimos } 12 \text { meses } \\
\text { (médico) }\end{array}$ & $\begin{array}{l}4,08(3,03-5,50) \\
3,92(3,37-4,56)\end{array}$ & $\begin{array}{l}2,81(1,89-4,16) \\
2,12(1,62-2,75)\end{array}$ \\
\hline \multicolumn{3}{|l|}{ Últimas 2 semanas } \\
\hline \multicolumn{3}{|l|}{ VPI } \\
\hline Não & 1 & 1 \\
\hline Sim & $3,60(2,38-5,42)$ & $2,90(1,38-6,09)$ \\
\hline
\end{tabular}

* valores em negrito indicam associação estatisticamente significante.

babilidade de relatar depressão. Observou-se que o tipo de violência mais prevalente no estudo foi a violência psicológica tanto em homens como em mulheres. Observamos ainda que mulheres, pessoas de cor branca e aqueles que procuraram mais os serviços de saúde tiveram maior probabilidade de relatar depressão.

O nosso estudo encontrou uma prevalência de 1,0\% de vítimas de VPI, sendo 1,58\% (IC95\% $=1,36-1,82)$ entre mulheres e 0,38\% (IC95\% =
0,26-0,54) entre homens. Este valor é considerado baixo quando comparado com a literatura nacional ${ }^{14,16}$. A subestimação na prevalência de VPI pode ser explicada por vários motivos. A principal explicação pode estar relacionada ao instrumento utilizado pela PNS para medir a VPI, que por não ser um questionário completo e validado para violência pode ter favorecido um viés de classificação não diferencial. Pesquisas que utilizaram questionário validado, englobam perguntas que descrevem os tipos de violência leve (empurrar, sacudir, estapear) e grave (chutar, queimar, usar faca ou arma de fogo), o que pode ter favorecido a compreensão de formas de violência pelo entrevistado ${ }^{16,17}$. No presente estudo, a pergunta sobre violência não apresentava exemplos gradativos conforme os estudos citados. Essa limitação pode ter levado à detecção principalmente dos casos mais graves, conforme literatura, ocasionando subestimação da prevalência ${ }^{18}$. Ademais, todos os grandes inquéritos com objetivo de vigilância em saúde utilizam instrumentos não validados que permitem uma aproximação de temas como a violência, e reconhecidas as limitações do instrumento possuem a grande vantagem de trabalharem com dados com representatividade populacional ${ }^{19,20}$.

Uma outra questão é que os temas relacionados à violência são considerados sensíveis e tendem a ser sub-relatados em entrevistas, talvez porque o entrevistado sinta-se constrangido em expor esse problema para pessoas desconhecidas. Por outro lado, incidentes relacionados à VPI podem ser minimizados e não ser considerados como agressões pelo entrevistado o que pode gerar o subrelato ${ }^{18}$. A delimitação de tempo para os últimos 12 meses, embora possa gerar viés de memória, é comum a outros inquéritos e por si só não poderia explicar a baixa prevalência. Por fim, um outro fator contribuinte poderia ser o fato que a PNS se baseou em amostra representativa da população brasileira e não em sub amostras de grupos vulneráveis como grande parte dos estudos de $\mathrm{VPI}^{21,22}$, e portanto, era de se esperar valores mais baixos em uma amostra com representatividade nacional.

Em relação ao sexo das vítimas, a prevalência de VPI foi maior entre as mulheres que entre os homens, o que se assemelha aos resultados reportados na literatura ${ }^{7,15}$. Quando analisamos o tipo de violência, a psicológica foi a forma mais frequente seguida da física, o que corrobora com resultados obtidos em outros estudos ${ }^{4,23,24}$.

O presente estudo mostrou que vítimas de VPI tiveram mais chances de relatar depressão 
em relação às pessoas que não sofreram VPI $(\mathrm{aOR}=2,90 ;$ IC95\% = 1,38-6,09), o que está em consonância com a literatura internacional ${ }^{13,25}$. A plausibilidade desta associação se baseia na depressão como um dos efeitos da violência entre parceiros íntimos ${ }^{5,6,26}$. Vale ressaltar, que dado o instrumento utilizado, o valor de OR observado pode estar subestimado, já que não podemos excluir a possibilidade de erro de classificação não diferencial, o que poderia reduzir a força de associação entre VPI e depressão ${ }^{27}$. Apesar dessa possibilidade, resultados utilizando instrumentos mais específicos para VPI e depressão observaram magnitude de associação semelhantes à do nosso estudo ${ }^{5,13}$.

A análise da depressão em relação ao sexo sugeriu que mulheres têm maiores probabilidades de desenvolver a doença $(\mathrm{aOR}=2,44 ; \mathrm{IC} 95 \%=$ 1,64-3,63), o que está de acordo com a literatu$\mathrm{ra}^{28,29}$. Analisando outras características que se associam à maior probabilidade de relatar depressão, observa-se maior probabilidade entre os indivíduos brancos quando comparados às demais cores, exceto indígenas. Esse resultado encontra-se em desacordo com boa parte da literatura que identifica populações pardas e negras com maiores prevalências de depressão ${ }^{28,29}$. Uma possível explicação para esse resultado seria o fato de que a depressão foi mensurada a partir do relato de diagnóstico por profissional de saúde e, no Brasil, existe grande desigualdade no acesso aos serviços de saúde; pessoas brancas possuem maior acesso quando comparadas às outras cores, e isto é bastante estabelecido na literatura ${ }^{30,31}$. Ainda que tenhamos realizado o ajuste para duas variáveis de acesso aos serviços de saúde, é possível que haja confundimento residual na associação entre cor e depressão, seja pelo acesso ou mesmo pela renda.

Os resultados desse estudo devem ser interpretados à luz de suas limitações. A limitação de temporalidade dos estudos transversais precisa ser considerada, sob o ponto de vista da ocorrência dos eventos analisados (VPI e depressão). A natureza do estudo não nos permite afirmar que a depressão ocorreu posteriormente à violência entre parceiros íntimos. Entretanto, a literatura dá suporte teórico à hipótese de que a VPI desencadeia sintomas depressivos e não o contrário. Nesse sentido, estudo longitudinal prévio já mostrou a direção de causalidade entre VPI e depressão ${ }^{32}$.

Outra limitação do estudo foi o fato de que o diagnóstico de depressão e o relato de VPI foram autorreferidos e não se utilizou questionários va- lidados, o que pode levar a viés de informação. Entretanto, todos os grandes inquéritos falham nesse aspecto porque visam investigar diversos aspectos de saúde diminuindo a especificidade das medidas estudadas ${ }^{33}$. Apesar disso, foi observada associação significativa entre VPI e depressão sugerindo que os erros de medida não prejudicaram os resultados. Outra questão relativa aos instrumentos utilizados, poderia ser o risco de viés de memória, pois as perguntas foram feitas se referindo aos 12 meses anteriores. Apesar disso, tanto a exposição quanto o desfecho são eventos relevantes, sobre os quais as pessoas possuem alta probabilidade de se lembrarem ${ }^{16}$.

As análises múltiplas para a associação entre VPI e depressão não foram estratificadas por sexo, pois a prevalência de vitimização por VPI, bem como a depressão em homens foi baixa e com isso, o modelo estatístico para o sexo masculino não alcançou convergência. Este fato nos impediu de realizar uma análise mais aprofundada para o sexo masculino e sugere a necessidade de mais estudos que possam investigar as associações entre homens.

Outra limitação foi que a PNS 2013 não dispunha de informações referentes à renda, por isso esse item não foi utilizado na análise dos dados. Porém, como forma de ajustar as análises para o nível socioeconômico, utilizou-se a escolaridade do indivíduo que pode ser considerada uma proxy da renda, já que pessoas com mais anos de estudo tendem a ter empregos com melhores salários e consequentemente ter maior renda ${ }^{34}$.

Este estudo transversal possui alguns pontos positivos. O primeiro é que o inquérito coletou dados de uma amostra representativa da população brasileira e teve alta taxa de resposta, o que minimiza o risco de viés de seleção. Além disso, o tamanho da amostra garantiu poder de análise para detectar as associações de interesse em um modelo ajustado por diversas variáveis. Por fim, a associação observada entre vitimização por VPI e depressão adiciona conhecimento à literatura existente e pode subsidiar práticas de vigilância em saúde no nível local, bem como políticas de saúde direcionadas a minimizar os efeitos da VPI.

Com os resultados deste estudo conclui-se que a depressão está associada à vitimização por parceiros íntimos o que tem implicações importantes no ponto de vista de saúde pública. Considerando a depressão como sendo efeito da VPI deve-se redobrar a atenção à causa da depressão para tratar não apenas as manifestações clínicas da doença, por meio de medicamentos, mas para que sejam trabalhadas suas causas, por exemplo, 
a violência. Também é importante salientar as mulheres como sendo a maioria das vítimas, mas não desconsiderando os homens nesse evento o que deve direcionar políticas públicas que minimizem a VPI, melhorem a identificação das vítimas dessa violência e direcionem para tratamento adequado no serviço de saúde. Para tal, outros estudos envolvendo VPI em ambos os sexos devem ser realizados para aprofundamento na temática.

\section{Colaboradores}

AN Silva trabalhou na concepção do artigo, na análise e interpretação dos dados, na sua revisão crítica e aprovou a versão a ser publicada. CM Azeredo contribuiu com a concepção do artigo, com a análise dos dados e a com a revisão final do texto. 


\section{Referências}

1. Breiding MJ, Basile KC, Smith SG, Black MC, Mahendra RR. Intimate Partner Violence Surveillance: Uniform Definitions and Recommended Data Elements, Version 2.0. Atlanta: National Center for Injury Prevention and Control, Centers for Disease Control and Prevention; 2015.

2. Bazargan-Hejazi S, Kim E, Lin J, Ahmadi A, Khamesi MT, Teruya S. Risk Factors Associated with Different Types of Intimate Partner Violence (IPV):An Emergency Department Study. J Emerg Med 2014; 47(6):710-720.

3. Organização Mundial da Saúde (OMS). Prevenção da violência sexual e da violência pelo parceiro íntimo contra a mulher: ação e produção de evidência. Washington: OMS; 2010.

4. Reichenheim ME, Moraes CL, Szklo A, Hasselmann MH, Souza ER, Lozana JA, Figueiredo V. The magnitude of intimate partner violence in Brazil: portraits from 15 capital cities and the Federal District. Cad Saude Publica 2006; 22(2):425-437.

5. Adeodato VG, Carvalho RR, Siqueira VR, Souza FGM. Qualidade de vida e depressão em mulheres vítimas de seus parceiros. Rev Saude Publica 2005; 39(1):108113.

6. Ferrari G, Agnew-Davies R, Bailey J, Howard L, Howarth E, Peters TJ, Sardinha L, Feder G. Domestic violence and mental health: a cross-sectional survey of women seeking help from domestic violence support services. Glob Health Action 2014; 7:25519.

7. Sugg N. Intimate Partner Violence: Prevalence, Health Consequences, and Intervention. Med Clin North Am 2015; 99(3):629-249.

8. Lagdon S, Armour C, Stringer M. Adult experience of mental health outcomes as a result of intimate partner violence victimisation: a systematic review. Eur J Psycho traumatol 2014; 5:1-12.

9. The World Bank. Brazil. [acessado 2016 Maio 3]. Disponível em: http://data.worldbank.org/country/brazil

10. Waiselfisz, JJ. Mapa da violência 2015: Mortes matadas por armas de fogo. Brasília: Unesco; 2015. [acessado 2016 Maio 25]. Disponível em: http://www.mapadaviolencia.org.br/pdf2015/mapaViolencia2015.pdf

11. Instituto Brasileiro de Geografia e Estatística (IBGE). Pesquisa Nacional de Saúde 2013: percepção do estado de saúde, estilos de vida e doenças crônicas. Rio de Janeiro: IBGE; 2014

12. Rothman KJ, Greenland S. Precision and validity in epidemiologic studies. In: Rothman KJ, Greenland S. Modern Epidemiology. Philadelphia: Lippincott-Raven; 1998. p. 115-134.

13. Coker AL, Davis KE, Arias I, Desai S, Sanderson M, Brandt HM, Smith PH. Physical and Mental Health Effects of Intimate Partner Violence for Men and Women. Am J Prev Med 2012; 23(4):260-268.

14. 14. D’Oliveira AFPL, Schraiber LB, França Júnior I, Ludermir AB, Portella AP, Diniz CS, Couto MT, Valença $\mathrm{O}$. Fatores associados à violência por parceiro íntimo em mulheres brasileiras. Rev Saude Publica 2009; 43(2):299-310.

15. Lindner SR, Coelho EBS, Bolsoni CC, Rojas PF, Boing AF. Prevalência de violência física por parceiro íntimo em homens e mulheres de Florianópolis, Santa Catarina, Brasil: estudo de base populacional. Cad Saude Publica 2015; 31(4):815-826.
16. Zaleski M, Pinsk I, Laranjeira R, Ramisetty-Mikler S, Caetano R. Violência entre parceiros íntimos e consumo de álcool. Rev Saude Publica 2010; 44(1):53-59.

17. Meekers D, Pallin SC, Hutchinson P. Intimate partner violence and mental health in Bolivia. BMC Womens Health 2013; 13:28.

18. Dias I. Estratégias de pesquisa qualitativa no estudo da violência na família. In: Esteves A, Azevedo J, organizadores. Metodologias qualitativas para as ciências sociais. Porto: Universidade do Porto; 1998. p. 29-39.

19. Sebert Kuhlmann AK, Foggia J, Fu Q, Sierra M. Intimate partner violence as a predictor of antenatal care service utilization in Honduras. Rev Panam Salud Publica 2017; 41:e104

20. Ogland E, Xu X, Bartkowski J, Ogland C. Intimate partner violence against married women in Uganda. J Fam Violence 2014; 29(8):869-879.

21. Ferreira MF, Moraes CL, Reichenheim ME, Verly JR E, Marques ES, Costa RS. Effect of physical intimate partner violence on body mass index in low-income adult women. Cad Saude Publica 2015; 31(1):161-172.

22. 22. Schraiber LB, D’Olivera AFPL, França-Júnior I, Diniz S, Portella AP, Ludermir AB, Valença O, Couto MT. Prevalência da violência contra a mulher por parceiro íntimo em regiões do Brasil. Rev Saude Publica 2007; 41(5):797-807.

23. Barros ÉN, Silva MA, Falbo-Neto GH, Lucena SG, Ponzo L, Pimentel AP. Prevalência e fatores associados à violência por parceiro íntimo em mulheres de uma comunidade em Recife/Pernambuco, Brasil. Cien Saude Colet 2016; 21(2):591-598.

24. Moura LBA, Gandolfi L, Vasconcelos AMN, Pratesi R. Violências contra mulheres por parceiro íntimo em área urbana economicamente vulnerável, Brasília, DF Rev Saude Publica 2009; 43(6):944-953.

25. Deyessa N, Berhane Y, Ellsberg M, Emmelin M, Kullgren $\mathrm{G}$, Ulf $\mathrm{H}$. Intimate partner violence and depression among women in rural Ethiopia: a cross-sectional study. Clin Pract Epidemiol Ment Health 2009; 5:8

26. Ahmadzad-Asl M, Davoudi F, Zarei N, MohammadSadeghi H, Rasoulian M. Domestic violence against women as a risk factor for depressive and anxiety disorders: findings from domestic violence house hold survey in Tehran, Iran. Arch Womens Ment Health 2016; 19(5):861-869.

27. Szklo M, Nieto FJ. Epidemiology: Beyond the basics. $2^{\text {nd }}$ ed. Sudbury: Jones and Bartlett Publishers; 2007.

28. Cunha RV, Bastos GAN, Duca GF. Prevalência de depressão e fatores associados em comunidade de baixa renda de Porto Alegre, Rio Grande do Sul. Rev Bras. Epidemiol. 2012; 15(2):346-354.

29. Almeida-Filho N, Lessa I, Magalhães L, Araújo MJ, Aquino E, Janes SA, et al. Social inequality and depressive disorders in Bahia, Brazil: interactions of gender, ethnicity, and social class. Soc. Sci. Med. 2004; 59(7):1339-1353.

30. Neri M, Soares W. Desigualdade social e saúde no Brasil. Cad Saude Publica 2002; 18(Supl.):77-87.

31. Ribeiro MCSA, Barata RB, Almeida MF, Silva ZP. Perfil sociodemográfico e padrão de utilização de serviços de saúde para usuários e não-usuários do SUS - PNAD 2003. Cad Saude Publica 2006; 40(11):1011-1022. 
32. Ridings LE, Beasley LO, Bohora SB, Daer JL, Owora A, Silovsky J. Longitudinal Investigation of Depression, Intimate Partner Violence, and Supports Among Vulnerable Families. J Interpers Violence 2016; 33(24):3749-3771.

33. Viacava F, Dachs N, Travassos C. Os inquéritos domiciliares e o Sistema Nacional de Informações em Saúde. Cien Saude Colet 2006; 11(4):863-869.

34. 34. Ramos, L. A desigualdade de rendimentos do trabalho no período pós-Real: o papel da escolaridade e do desemprego. Economia Aplicada 2007; 11(2):281301 .

Artigo apresentado em 18/09/2016

Aprovado em 19/10/2017

Versão final apresentada em 21/10/2017 